\title{
PSYCHOPHYSIOLOGY
}

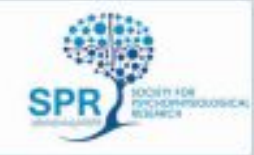

\section{Two distinctive stages of novelty processing revealed by high-density EEG based source imaging}

\begin{tabular}{|c|c|}
\hline Journal: & Psychophysiology \\
\hline Manuscript ID & PsyP-2021-0152 \\
\hline Wiley - Manuscript type: & Special Issue \\
\hline $\begin{array}{r}\text { Date Submitted by the } \\
\text { Author: }\end{array}$ & 18-Mar-2021 \\
\hline Complete List of Authors: & $\begin{array}{l}\text { Tsang, Eric; University of Hong Kong, Faculty of Education } \\
\text { Sun, Rui; University of Hong Kong, Laboratory of Neuroscience for } \\
\text { Education } \\
\text { Niu, Xueyan; University of Hong Kong } \\
\text { Fung, Wei Yan Renee; University of Hong Kong } \\
\text { Tang, Akaysha; University of Hong Kong, Faculty of Education }\end{array}$ \\
\hline Keywords: & $\begin{array}{l}\text { P300, Visual Oddball, Time Frequency analysis, Theta frequency, Second } \\
\text { Order Blind Identification, EEG < Methods }\end{array}$ \\
\hline Abstract: & $\begin{array}{l}\text { Novelty detection is an evolutionarily significant and ancient function as } \\
\text { well as a relatively stable function whose early life status marks for long- } \\
\text { term developmental outcomes and predicts a range of adult functions. } \\
\text { While various brain regions have been shown to respond to } \\
\text { environmental novelty, how different brain regions coordinate in novelty } \\
\text { related information processing remains under-explored. Here using a } \\
\text { combination of high-density EEG, second order blind identification } \\
\text { (SOBI), and a standard visual oddball task, we test, in humans, a two- } \\
\text { stage novelty processing hypothesis which states that two distinct stages } \\
\text { of novelty processing exist, one involves early-occurring domain-specific } \\
\text { neural activity in the sensory processing areas of the brain and the other } \\
\text { involves later-occurring domain-general neural activity involving brain } \\
\text { regions beyond the sensory cortices. We found that: (1) a significant } \\
\text { Novelty effect (oddball effects) not only in the SOBI-recovered Late } \\
\text { component (P300 component) but also in the Early component (N150 } \\
\text { visual) offering first EEG evidence for oddball effect in the sensory } \\
\text { domain; (2) a significant Stage (Early vs Late) by Frequency (delta, } \\
\text { theta, alpha, beta, and gamma) interaction effect indicating two } \\
\text { functionally dissociable mechanisms underlying novelty detection; (3) a } \\
\text { significantly shorter latency in odd-ball related theta power increase in } \\
\text { the Early visual than in the late P300 component. These results not only } \\
\text { offer support for the two-stage novelty processing theory but also } \\
\text { provide new evidence for an early involvement of theta power increase in } \\
\text { the novelty processing. }\end{array}$ \\
\hline
\end{tabular}




\section{SCHOLARONE ${ }^{\text {"W }}$ Manuscripts}

Psychophysiology 


\section{Impact Statement}

Our findings provided new concrete evidence that human novelty processing consists of two distinct stages: a domain-specific Early component in the visual sensory region and a domaingeneral Late component in the centrofrontal region. This is the first report and isolation of novelty elicited theta frequency in visual sensory region. This Early theta frequency modulates that of the Late component and has behavioral significance. 
Two distinctive stages of novelty processing revealed by high-density EEG based source imaging

\section{Short title:}

Two distinctive stages of human novelty processing

Eric W. Tsang ${ }^{1 *}$, Rui Sun ${ }^{1}$, Xueyan Niu ${ }^{1}$, Wei Yan Renee Fung ${ }^{1}$, Akaysha C. Tang ${ }^{1,2}$

${ }^{1}$ The Laboratory of Neuroscience for Education, The University of Hong Kong, Hong Kong

${ }^{2}$ The Mind Research Network, Albuquerque, NM. USA

*Correspondence to: Eric W. Tsang; The Laboratory of Neuroscience for Education, Faculty of Education, The University of Hong Kong, Hong Kong

Tel: 852-39170389; E-mail: ew_tsang@yahoo.com

\section{ORCiDs}

Eric W. Tsang https://orcid.org/0000-0002-6333-9584

Number of pages: 22 excluding references

Number of figures: 5

Number of tables: 2

Number of words in Abstract: 235

Number of words in Impact Statement: 63 


\begin{abstract}
Novelty detection is an evolutionarily significant and ancient function as well as a relatively stable function whose early life status marks for long-term developmental outcomes and predicts a range of adult functions. While various brain regions have been shown to respond to environmental novelty, how different brain regions coordinate in novelty related information processing remains under-explored. Here using a combination of high-density EEG, second order blind identification (SOBI), and a standard visual oddball task, we test, in humans, a twostage novelty processing hypothesis which states that two distinct stages of novelty processing exist, one involves early-occurring domain-specific neural activity in the sensory processing areas of the brain and the other involves later-occurring domain-general neural activity involving brain regions beyond the sensory cortices. We found that: (1) a significant Novelty effect (oddball effects) not only in the SOBI-recovered Late component (P300 component) but also in the Early component (N150 visual) offering first EEG evidence for oddball effect in the sensory domain; (2) a significant Stage (Early vs Late) by Frequency (delta, theta, alpha, beta, and gamma) interaction effect indicating two functionally dissociable mechanisms underlying novelty detection; (3) a significantly shorter latency in odd-ball related theta power increase in the Early visual than in the late P300 component. These results not only offer support for the two-stage novelty processing theory but also provide new evidence for an early involvement of theta power increase in the novelty processing.
\end{abstract}

Key words: P300, Visual Oddball, Time Frequency analysis, Theta frequency, Second Order Blind Identification, EEG 


\section{Introduction}

Novelty detection is an evolutionarily well-conserved function for it is critical for both detecting and avoiding dangers and learning about and adapting to environmental changes (Dasgupta, Sheehan, Stevens, \& Navlakha, 2018; Sokolov, 1963). In animal model systems, the computation of novelty appears to involve both domain-specific sensory systems and domain-general systems (Perez-Gonzalez, Malmierca, \& Covey, 2005) where contextual information converge from distinct domains of sensory processing to domain general systems (Jeewajee, Lever, Burton, O'Keefe, \& Burgess, 2008) that control the dynamic release of various neuromodulators (Schultz, 1998).

In humans, in part due to the feasibility of measuring techniques, parallel invasive studies are rarely available, but only during neurosurgeries where control over the experimental settings are limited (Lachaux, Axmacher, Mormann, Halgren, \& Crone, 2012). Adaptation of single unit activity has been recorded from awake human patient (Jacobs, Kahana, Ekstrom, \& Fried, 2007) and changes in hippocampal theta power associated with learning of a new environment has been observed through the use of non-invasive scalp EEG (Cohen, Morin, \& Stern, 2018). Unique to the human studies, majority of evidence regarding how the human brain processes novelty information has been mainly from brain imaging studies using fMRI and with EEG using a few well studied experimental paradigms (Ranganath \& Rainer, 2003). For example, fMRI studies have revealed a variety of brain regions ranging from the early sensory processing areas to frontal regions and subcortical regions showing differential BOLD signal to changes in stimulus novelty (Clark, Fannon, Lai, Benson, \& Bauer, 2000; Gur et al., 2007). Due to fMRI temporal resolution on the order of seconds, it cannot be determined whether the inferred neural response to novelty in the early sensory area occurs prior to the activity associated with the later central 
processing. In contrast, EEG studies using visual oddball task have generated a large body of literature on a novelty-related potentiation of a large amplitude response of the event related potentials that typically occur around $300 \mathrm{~ms}$ from the stimulus onset (hence the name of P300) (Friedman, Cycowicz, \& Gaeta, 2001; Sutton, Braren, Zubin, \& John, 1965). However, due to volume conduction, the ERPs measured by the scalp electrodes reflect an unknown mixture of neuronal signals from different brain regions as well as non-neuronal signals associated with various artefact, i.e. an electrode located over the frontal cortex may in part reflect signals from non-frontal regions and vice versus.

Interestingly, previous studies using the low temporal-resolution fMRI consistently reported increased hemodynamic responses for visual novel stimuli in sensory regions including the bilateral occipital and posterior temporal cortices in addition to the typically reported frontal regions (Ardekani et al., 2002; Clark et al., 2000; Gur et al., 2007; Kieh, Laurens, Duty, Forster, \& Liddle, 2001; Linden et al., 1999). The high temporal resolution EEG studies indicated that visual novelty induced alpha frequency desynchronization (ERD) at bilateral occipital cortices which was suggested as indication of cortical activation and this alpha ERD was later spread forward to more anterior regions for higher cognitive processes (Peng, Hu, Zhang, \& Hu, 2012; Peng, Hu, Mao, \& Babiloni, 2015). Together, these findings lead to the hypothesis that there exist at least two stages of novelty processing, one involving a rapid domain-specific computation of novelty and the second a relatively slower domain-general computation of novelty. For visual novelty detection, this early stage involves the posterior sensory region such as the occipital and posterior temporal cortices and the later stage involves the centrofrontal regions typically reported as the P300 component. 
However, the temporal spatial relationship between these stages of visual novelty processing are not well understood. The aim of this study is to apply Second-order blind identification (SOBI) for the study of these two stages of visual novelty processing. In this study, we investigated visual novelty processing using the SOBI technique with a particularly interest in early sensory processing. This would facilitate the advancement of the current understanding of network activities involving visual novelty processes.

\section{METHOD}

\subsection{Participants}

Thirteen right-handed young and healthy subjects (6 males and 7 females), aged between 19 and 33 years $(26.50 \pm 4.48$, mean $\pm \mathrm{SD})$, volunteered to participate in this experiment. All participants reported normal or corrected-to-normal vision and without histories of neurological or psychological disorder. Ethics approval of this study was obtained from the Human Research Ethics Committee of the University of Hong Kong. This study was conducted in accordance with the principles of the Helsinki declaration. All subjects gave written informed consents before their participation.

\subsection{Experimental procedures}

Participants were studied individually. They were seated in a comfortable chair in front of a computer screen in a lighted room. The eyes-to-screen distance was $64 \mathrm{~cm}$. All participants performed a standard visual oddball task (Malik \& Amin, 2017) where two center-field color rectangles were used as frequent and rare stimuli. The frequent stimulus was a blue rectangle and the rare stimulus was a red rectangle. The size of both frequent and rare stimuli was the same 
(7.25 $\mathrm{cm}$ in height and $9.2 \mathrm{~cm}$ in width). For each trial, a frequent or rare rectangle was presented for $500 \mathrm{~ms}$ with an inter-trial-interval randomly ranging from 2033 to $4016 \mathrm{~ms}$ between trials. The task required the participants to press the "spacebar" on the keyboard when a rare stimulus appeared and 'not to respond' for a frequent stimulus. The participants were instructed to respond as quickly as possible while avoiding errors. Reaction time and correct target (rare stimulus) detection were recorded. The task contained 250 trials, in which 50 trials contained rare stimulus and 200 trials contained frequent stimulus that is $20 \%$ of the trials contained a rare stimulus and $80 \%$ contained a frequent stimulus. The visual oddball task was conducted by Eprime 2 software (Psychology Software Tools, Pittsburgh, PA, USA). The duration of the task was approximately 15 minutes.

\subsection{EEG recording and pre-processing}

High-density EEG signals were continuously recorded from the participants during the oddball task using a 128-channel active $\mathrm{Ag} / \mathrm{AgCl}$ electrode cap (actiCAP) based on the international 1020 system connected to an actiCHamp battery-supplied active channel amplifier (Brain Products GmbH, Munich, Germany). All electrodes were not physically referenced and a single ground electrode was located at the forehead. EEG was bandpass filtered between 0.1 and $200 \mathrm{~Hz}$. Impedance was maintained below $5 \mathrm{k} \Omega$ and the sampling rate was $1000 \mathrm{~Hz}$.

Oddball trials with incorrect or no response as well as those with latencies later than 3000 ms were excluded from the data analysis. The SOBI Blind Source Separation algorithm was applied directly to continuous EEG data to perform component analysis and artifact separation. Unlike conventional analysis of continuous EEG data which requires ocular artifact correction and visually identified artifact rejection, SOBI has the ability to separate the artifact effectively and does not require the conventional EEG pre-processing procedure (A. C. Tang, Pearlmutter, 
Malaszenko, Phung, \& Reeb, 2002; A. C. Tang, Sutherland, \& McKinney, 2005). Tang et al (2005a) provides a detailed description of SOBI processing.

\subsection{Second-order blind identification (SOBI)}

SOBI (Belouchrani, Abed-Meraim, Cardoso, \& Moulines, 1997) is a signal processing technique that can be used to facilitate source analysis from EEG (Joyce, Gorodnitsky, \& Kutas, 2004; A. C. Tang, Liu, \& Sutherland, 2005; A. C. Tang, Sutherland, et al., 2005) and MEG data (Mackert, Wubbeler, Leistner, Trahms, \& Curio, 2001; A. C. Tang, Pearlmutter, Malaszenko, \& Phung, 2002; A. C. Tang, Pearlmutter, Malaszenko, Phung, et al., 2002; Akaysha C. Tang, Pearlmutter, Zibulevsky, \& Carter, 2000). This method enables both model-free extraction of electric or magnetic signals associated with neuronal activity at specific brain locations and simultaneous isolation of large-amplitude ocular and sensor artifacts when given continuously recorded EEG or MEG data. Therefore, this method may be particularly useful for data analysis in cognitive neuroscience to separate scalp signals which do not map directly to signals arising from functionally specific brain areas but retain network related signals that are functionally related to specific brain regions. From a practical point of view, SOBI's performance is robust across participants (A. C. Tang, Sutherland, et al., 2005) and across repeated measures of trials over various lengths of time intervals (A. C. Tang, Liu, et al., 2005; A. C. Tang, Sutherland, et al., 2005).

\subsection{Identification of candidate SOBI components for early visual and late P 300}

We identified SOBI components that corresponded to the early visual and late P300 according to a similar set of spatial and temporal criteria described previously (A. C. Tang, Liu, et al., 2005;

A. C. Tang, Sutherland, et al., 2005). Briefly, using stimulus-triggered averages from each 
individual subject, we obtained a subset of components from the 128 total recovered components that displayed early visual and late P300 ERPs, which were defined as positive or negative peaks at latencies between 50 and $250 \mathrm{~ms}$ for early visual component and between 200 and $700 \mathrm{~ms}$ for late P300 component. The peaks of these pre-selected early visual and late P300 components would have similar latencies in rare and frequent conditions. Moreover, the peaks displayed oddball effects which are greater amplitudes in the rare compared to the frequent conditions. The number of the pre-selected early visual and late P300 components were further reduced based on their activation patterns in the sensor space projections, as shown in scalp current source density (CSD) maps. The CSD maps were computed from the scalp potential maps as the second spatial derivative of the voltage distribution and are better at revealing visually the underlying generators than the potential maps (Lagerlund, 1999). To be candidates of the early visual or late P300 components, the CSD maps must show the characteristic pattern of activation. The prototype spatial patterns for early visual components were posterior visual sensory regions including the bilateral occipital, posterior parietal, and posterior temporal cortices. The spatial patterns of the late P300 components would involve the medial central regions and may partly encompass the frontal or the parietal regions. Only candidate components that met both the ERP and CSD map criteria were chosen. Through visual inspection, we selected one early visual and one late P300 components for each subject from the candidate components based on their best meeting the ERP and CSD map criteria without ambiguity (Fig. 1).

\subsection{Frequency domain analysis of novelty effects}

Single-trial frequency spectrums were obtained with Fast Fourier transformation to the source ERPs of the selected early visual and late P300 components from 0 to $1000 \mathrm{~ms}$ from each subject 
in both frequent and rare conditions, which were followed by averaging the single trial spectrums to obtain the average frequency spectrums of early visual and late P300 components for each subject in frequent and rare conditions. The average frequency spectrums of both components were divided into five frequency bands including Delta: 1-3 Hz, Theta: 4-7 Hz, Alpha: 8-15 Hz, Beta: 16-31 Hz, and Gamma: 32-60 Hz. These cut-off ranges were chosen based on their proven suitability for the analysis of global resonance phenomena of brain structures (Basar, BasarEroglu, Demiralp, \& Schürmann, 1995).

Visual novelty effects were defined as the differences in spectra power in each frequency band elicited by rare from those of frequent stimuli, which were calculated by the subtraction of spectra power of rare by those of frequent stimuli in both early visual and late P300 components. One-sample t-tests were used to examine the statistical significance of novelty effect in each frequency band in both components.

\subsection{Event-related time frequency analysis}

To examine the temporal domain information of frequency bands following frequency spectrum analysis, we performed event-related spectral perturbation (ERSP) analysis to reveal the changes in frequency power under three conditions (frequent, rare, and rare - frequent). We selected the range of ERSP between 3 and $40 \mathrm{~Hz}$ where the majority of EEG related spectral power were observed. The ERSP time interval was from -200 and $800 \mathrm{~ms}$ where $0 \mathrm{~ms}$ denoted the onsets of the stimuli. ERSP transforms were computed by the Wavelet Toolbox in Matlab (The MathWorks, Inc., Natick, Masachuetts, United States) using Complex Morlet wavelets with the central frequency of $1 \mathrm{~Hz}$ and the decay of 1 and normalized by subtracting the power of baseline (-200 to $0 \mathrm{~ms}$ ). The use of $1 \mathrm{~Hz}$ central frequency produced the maximal temporal 
resolution for the wavelet transformation (Allen \& MacKinnon, 2010), which was very useful for our purpose of investigating novelty elicited frequency effects especially the early theta frequency.

\subsection{Dipole modelling of the Early visual component}

In order to determine the source of the Early component, we performed dipole source modelling in all 13 subjects using Brain Electric Source Analysis (BESA 6.1), from which the fitting was calculated from the surface CSD dipoles within a standardized brain between the recorded (Data) and calculated (Model) electric field distribution.

The averaged ERPs generated by the Early visual component from each subject (Fig, 1) was used. Only rare trials were analysed. The baseline interval used was the whole interval from -200 to $800 \mathrm{~ms}$. For dipole modelling, the latency interval was from 50 to $250 \mathrm{~ms}$ post-stimulus. An age-appropriate template of adult head model was used and a symmetric constrain was applied to the bilateral sources at the visual cortex. One pair of symmetric dipoles was first applied to the posterior visual areas in the initial analysis. After the first round of fitting, we examined the fitting between the Data and Model and the amount of residual variance (RV). An additional pair of dipoles would be applied when the fitting between Data and Model was less than optimal (goodness of fit (gof) $<90 \%$ ) and substantial RV $(>10 \%$ ) was unaccounted for by the Model. An ideal fitting was considered when the fitting between the Data and Model was ideal (gof $>90 \%)$ and minimal RV $(<10 \%)$ in the cortical map was observed. The dipole coordinates were exported in the Talairach format measured in millimetres. The software, Talairach Client, was applied to determine the location of each pair of individual dipoles in each subject. Cube Range ( $\pm 5 \mathrm{~mm})$ was applied and results with hits above 100 were used. 


\subsection{Statistical analyses}

The amplitudes of the early and late components in each subject were normalized so that the ERP and frequency data of each component have the unit of microvolt. The normalization process first involved the projection of amplitudes in microvolt of each component back to the 128 scalp electrodes. This was followed the identification of the electrode in each component with the highest amplitude in microvolt. This highest amplitude in microvolt in each component was used to multiply the amplitudes of the frequency bands and ERPs during the normalization process.

To test for the novelty effects, we first computed the differences in spectral power of the five frequency bands between rare and frequent trials. Due to large differences in spectral powers were noted between the delta and the higher frequency bands such as the beta and gamma frequency bands, we further normalized the spectral powers of the five frequency bands by cubic root transformation to reduce the differences in spectral powers. This was followed by the examination of novelty effects associated with the early visual and late P300 components by a two-way repeated measures ANOVA with component type (early visual versus late P300) and frequent band (delta, theta, alpha, beta and gamma) as within-subject factors. Post hoc analyses were performed by paired sample t-tests to examine the interaction effects of different frequency bands in the two components.

For statistical analyses of the ERSP data, we compared the peak latencies and powers of the theta and alpha frequency bands in the early and late components. We applied paired sample t-tests to compare the differences in peak latencies between the theta and alpha bands in the early and late components and followed by comparing the peak latency differences in the theta and alpha bands between the early and late components. Moreover, we examined the differences in 
peak power of the theta and alpha bands between the two components. The results of the paired sample t-tests were further confirmed with those of the nonparametric analysis using the Wilcoxon Signed Ranks Test (WSRT). To establish the behavioural relationship of the novelty elicited theta bands, we performed Pearson correlation analyses using the latencies of Early or Late peak theta bands as independent variables and the subject's reaction time to button press as the dependent variable. The effect sizes of statistically significant ERPs, frequency bands, and ERSP analyses as revealed by paired t-tests were estimated by partial $\eta^{2}$ calculated by repeated measure ANOVA.

\section{RESULTS}

\subsection{Time domain analysis.}

We were able to identify an Early visual and a Late P300 component in all participants (Fig. 1). These two component types show distinct patterns of waveforms with the ERPs reaching peaks on average at Early: $126 \pm 9 \mathrm{~ms}$ and Late: $304.38 \pm 10 \mathrm{~ms}$ for frequent conditions and Early: $127.54 \pm 7 \mathrm{~ms}$ and Late: $358.08 \pm 9 \mathrm{~ms}$ (Mean \pm SEM) for rare conditions and the peak latencies differ significantly [Frequent: $\mathrm{t}(12)=-11.26, \mathrm{P}<0.0001$, partial $\eta^{2}=0.91$; Rare: $\mathrm{t}(12)=-17.69, \mathrm{P}<$ 0.0001, partial $\left.\eta^{2}=0.96\right]$. A significant oddball effect (Rare vs Frequent) on the ERP peak amplitude was found in not only the Late components (Rare: $9.09 \pm 1 \mu \mathrm{V}$, Frequent: $4.87 \pm 0.65$ $\mu \mathrm{V})\left[\mathrm{t}(12)=6.87, \mathrm{P}<0.0001\right.$, partial $\left.\eta^{2}=0.8\right]$, replicating the previously known effect, but also the Early components (Rare: $6.37 \pm 0.77 \mu \mathrm{V}$, Frequent: $4.43 \pm 0.59 \mu \mathrm{V}$ ) [t(12) $=4.48, \mathrm{P}=0.001$, partial $\left.\eta^{2}=0.63\right]$. The ERPs in higher resolution and their peaks of both components are provided in Supplementary Fig. 1 and 2. 


\subsection{Frequency domain analysis.}

Fig. 2AB provide comparison between the Freq and Rare conditions in power across delta, theta, alpha, beta, and gamma bands (2AB for Early and Late components respectively). Fig. 2C contrasts between the Early and Late components in oddball effects (Rare-Frequent). Central to our hypothesis of distinctive mechanisms underlying novelty processing is the observation that Early and Late components showed distinct patterns of neural activities across different frequency bands (Fig. 2C) as indicated by the significant Comp x Freq interaction effect $(\mathrm{F}(4$, $48)=4.36, P=0.004$, partial $\left.\eta^{2}=0.27\right)$. The main effects of Comp and Freq were also significant [Comp: $F(1,12)=13.62, P=0.003$, partial $\eta^{2}=0.53$; Freq: $F(4,48)=24.67, P<0.0001$, partial $\eta^{2}=$ 0.67].

With the protection of the statistically significant interaction effect, we performed post hoc paired t-tests to further characterize the Early vs Late component difference across each of the five pairs of frequency bands. The Early and Late components differ in their oddball effects in the delta, theta, alpha, beta (marginal) bands [Fig. 2C: Delta: $\mathrm{t}(12)=2.95, \mathrm{P}=0.012$, partial $\eta^{2}=$ 0.42; Theta: $\mathrm{t}(12)=3.19, \mathrm{P}=0.008$, partial $\eta^{2}=0.46$; Alpha: $\mathrm{t}(12)=5.90, \mathrm{P}=0.0001$, partial $\eta^{2}=$ 0.74; Beta: $\left[\mathrm{t}(12)=1.92, \mathrm{P}=0.08\right.$, partial $\left.\eta^{2}=0.24\right]$. While the oddball effects are in the same direction (increase in power) for both components in the delta and theta bands, the effects are in the opposite direction in the alpha, beta, and gamma bands with the Early component showing the general trend of power decrease.

Further follow up with one sample t-tests were performed to determine whether the oddball effect can be detected (rare - frequent) for each component and in each frequency band. For the Early component, in addition to the expected significant reduction in alpha power [t(12) $=-10.44, \mathrm{P}<0.0001]$, we found a surprising significant oddball effect in theta power increase 
$[\mathrm{t}(12)=2.73, \mathrm{P}=0.018]$. For the late $\mathrm{P} 300$ component, oddball effects were found in both the delta $[\mathrm{t}(12)=8.99, \mathrm{P}<0.0001]$ and theta $[\mathrm{t}(12)=27.20, \mathrm{P}<0.0001]$. No other effects were found.

\subsection{Time-frequency analysis.}

To determine the difference in precise timing of theta power increase between the Early and Late components, we made time-frequency plots for the Early and Late components (Fig. 3 A-F: group average; G-L: an individual example). Table 1 presents the peak latencies and power of theta and alpha frequency bands of the early component in each subject. We found that the Early component has a significant shorter latency in peak theta power increase (an oddball effect) (Fig. 3C) than the Late component (Fig. 3F) (Early: 205/196 \pm 25 ms; Late: 344/334 \pm 16 ms; $($ Mean/Median \pm SEM $)\left[\mathrm{t}(12)=-7.76, \mathrm{P}<0.0001\right.$, partial $\eta^{2}=0.83$; Wilcoxon's: $\mathrm{Z}=2.97, \mathrm{P}=$ 0.003). The latency of peak alpha power suppression was 546/ $623 \pm 50 \mathrm{~ms}$ in the Early component which is significantly later than the above described theta power increase (Fig. 3C) $\left[\mathrm{t}(12)=-6.91, \mathrm{P}<0.0001\right.$, partial $\eta^{2}=0.80$; Wilcoxon's: $\left.\mathrm{Z}=-3.11, \mathrm{P}=0.002\right]$. The above mentioned latency of peak theta power increase in the Late component was significantly earlier than the peak alpha power suppression in the Late component $(494 / 496 \pm 51 \mathrm{~ms})$ (Fig. 3F) $\left[\mathrm{t}(13)=-2.73, \mathrm{P}=0.018\right.$, partial $\eta^{2}=0.38$; Wilcoxon's: $\left.\mathrm{Z}=2.20, \mathrm{P}=0.028\right]$. Furthermore, Pearson correlation revealed that the latency of oddball induced peak theta power increase in the Early component was significantly correlated with subjects' reaction time of button press $(\mathrm{r}=0.6, \mathrm{P}=$ 0.032) and the latency of oddball induced peak theta power increase in the Late component $(\mathrm{r}=$ $0.7, \mathrm{P}=0.008)$. The latencies of the oddball induced peak alpha power suppression in the Early component, or the peak theta power increase and peak alpha power suppression in the Late 
component were not correlated with subjects' reaction time. Fig. 4 presents the scatterplots for the correlation analyses.

\subsection{Dipole modelling of the Early visual component}

Dipole modelling analyses revealed that the CSD dipoles between Data and Model were very well fitted in the Early visual components for all subjects with group average goodness of fits (gof): $95 \pm 1 \%$ and RV: $5 \pm 1 \%$. The solutions of equivalent current dipoles of the Early visual component in all subjects consisting of 1 pair in 7 subjects. 2 pairs in 3 subjects, 3 pairs in 1 subject, and 4 pairs in 1 subject. Each subject has 1 pair of symmetrically placed dipole sources within the secondary visual cortex (Fig. 5, Table 2). The average Talairach coordinates; x: $28 \pm$ 3; y: $-82 \pm 3 ; z: 7 \pm 5 \mathrm{~mm} ;(\mathrm{N}=13)$. Talairach Client revealed that the sources of the Early visual components in all individual thirteen subjects were in the secondary visual cortex (Brodmann's areas 18 and 19).

\section{DISCUSSION}

This work is motivated by the belief that understanding novelty detection and the subsequent processing of novelty information is central to the understanding of learning and adaption. In a high-density EEG study of a two stimuli color visual oddball task, we performed time-domain, frequency-domain, and time-frequency analysis of two components, an early visual component and a late P300 component, derived by a BSS algorithm - SOBI — to test the hypothesis that novelty processing consists of at least two stages, an early stage that is sensory domain-specific and a later stage that is domain general involving brain regions beyond the sensory cortices. We 
made the following observations: the Early and Late components (1) both showed significant oddball effects and with the Early component showing significantly shorter ERP peak latencies ( $230 \mathrm{~ms}$ ) than the Later component; (2) showed distinct patterns of novelty elicited frequencydomain response in delta, theta, and alpha frequency bands, but in particular the theta band modulates both Early and Late components during novelty detection (3) showed significantly different latencies in peak theta power increase with the Early component's oddball response being significantly earlier than the Late component ( $\sim 150 \mathrm{~ms})$. These findings support the hypothesis of two distinct stages of novelty processing and offer the critical temporal information to disambiguate the interpretation of previously reported fMRI evidence for parallel activation of occipital cortices and other non-sensory cortices.

\subsection{Why two stages of novelty processing?}

If one is to build a system that must adapt to a changing environment, then this system must be able to rapidly detect changes or novelty in the environment, i.e. detecting an increase in entropy or uncertainty, and then make necessary changes to the system to better predict its future occurrence. So one way of building this system is to build detectors in different sensory domains that uses local domain-specific networks to compute novelty signals within that domain (Bassett, Yang, Wymbs, \& Grafton, 2015; Mohr et al., 2016), which in turn can be sent to the secondstage action-censors which in turn can broadcast novelty signals to all sensory domains to enable the incorporation of contextual information into new memory for this novel event (Shine et al., 2016). Reasonable candidates for the first stage processing would be the primary and secondary sensory cortices and its corresponding thalamic input regions and for the second stage processing could include hippocampus, frontal cortex, and other subcortical structures that control for the 
diffused release of various neuromodulators and their innervation of large cortical networks, critical for the neural dynamics of learning and memory (Shine, 2019).

Although previous works from fMRI studies of visual and auditory oddball tasks and the EEG alpha suppression by oddball stimuli (Peng et al., 2012; Peng et al., 2015; Pfurtscheller, Neuper, \& Mohl, 1994) are all consistent with these two sets of brain regions, they do not provide the critical information to allow the assessment of temporal order by which the two types of novelty processing take place. By successfully extracting the two components, one displaying a temporally earlier novelty responses than the other in both the time and frequency domains, the present study offered novel evidence in support of the two-stage processing of novelty information.

\subsection{Benefit of source-domain analysis}

This study presented the first evidence that a visual oddball effect can be detected by early visual processing areas of the brain with a very short latency of approximately $130 \mathrm{~ms}$ from the stimulus onset. This is not surprising for it would be more surprising to claim that novelty detection needs as much as $300 \mathrm{~ms}$ or more to complete. This finding extended our current understanding of neural mechanisms underlying novelty processing as tested in the visual oddball task. Then why, given the very large body of literature on the visual oddball tasks, wasn't such an observation made in the past?

One likely explanation is that most of the past work was done in the sensor-domain analysis and because of volume conduction, the recorded signals by a given sensor is a mixture from many sources who have different time courses of variations. As such, sensor-domain signals should in principle have relatively poor signal to noise ratio. Empirical work has 
demonstrated that indeed by moving into source-domain using SOBI, one can significantly increase single-trial ERP's signal to noise ratio such that (1) better single-trial ERP classification performance can be achieved (A. C. Tang, Pearlmutter, Malaszenko, \& Phung, 2002); (2) very weak and previously deemed unreliable signals associated with ipsi-lateral responses can be reliably detected with 100\% success rate (Sutherland \& Tang, 2006); (3) single-trial visual ERPs can be measured even in the presence of concurrent continuous free eye movement (A. C. Tang et al., 2006).

\subsection{Rapid detection of stimulus novelty via early theta power increase}

The time-frequency analysis of the Early component's response to the Frequent and Rare stimulus conditions further revealed that the early oddball effect is also expressed in the frequency domain as an increase in theta power. The onset of this theta power increase was as early as $\sim 30 \mathrm{~ms}$ and reaching the peak power increase at approximately $200 \mathrm{~ms}$. This Early component's theta power increase occurred long before the onset of the theta power increase in the Late P300 component $(\sim 70 \mathrm{~ms})$ and peak at $\sim 340 \mathrm{~ms}$, therefore cannot be a result of that late theta power increases and must be computed by mechanisms independent from the Late P300 component. In terms of functions, this Early component's theta power increase was correlated with subjects' behavioural response indicating that this early domain specific processing is associated with rapid behavioural response to novel stimuli which is particularly adaptive to environmental challenges. In addition, the latency of Early peak theta power not only lead that of the Late peak theta power, but they showed significant correlation to each other. This implicated that the Early component provide domain specific sensory information for the computation of the 
domain general, Late P300 component, during novelty processing in line with the network theory of segregation and integration of cognitive processing (Mohr et al., 2016; Shine et al., 2016).

Given that the onset of Early theta precedes that of the Late P300 component, what is the origin of this Early component theta? Our finding from dipole modelling suggest the Early visual component is generated at the secondary visual cortices. The local generator at the sensory cortex has also been demonstrated by a study which showed theta band coherence during working memory task recorded by implanted electrodes in the occipital and parietal cortices (Raghavachari et al., 2006). An increase in the theta coherence were most often recorded by pairs of electrodes located in the occipital, parietal, and temporal cortices in comparison to the frontal cortex. The synchronization of theta band power was also in the visual cortices in monkeys using intracortical electrodes (Lee, Simpson, Logothetis, \& Rainer, 2005; Rainer, Lee, SIMpson, \& Logothetis, 2004). The possibility of a locally generated theta band oscillation is further supported by in vitro electrophysiology studies in which theta band oscillation can be induced in cortical local networks under appropriate pharmacological conditions (Blatow et al., 2003; Flint \& Connors, 1996; Silva, Amitai, \& Connors, 1991). The present study offers the first evidence from a non-invasive study of humans to support the likely existence of a locally generated theta power increase as a result of novelty detection.

It is interesting to note that the same Early component also exhibits delayed oddball effect of alpha power suppression, a phenomenon reported previously by a number of studies. These studies show that using LORETA, the novelty elicited alpha power reduction was generated at the occipital cortices at a onset latencies of $\sim 420 \mathrm{~ms}$ (Peng et al., 2012; Peng et al., 2015; Pfurtscheller et al., 1994). Therefore, our finding of both a theta power increase and an 
alpha power decrease due to oddball effect not only replicates results from these previous studies but also serves to confirm the early sensory processing origin of this Early visual component.

\subsection{Conclusions and Future Work}

We provided evidence from the visual domain to support a hypothesis of two-stage novelty processing and to show the involvement of an early theta oscillation in visual novelty detection. Future work can take several directions, including testing the hypothesis in other sensory domains, such as auditory, somatosensory, etc; testing the hypothesis using a variety of learning and memory paradigms. 


\section{References}

Allen, D. P., \& MacKinnon, C. D. (2010). Time-frequency analysis of movement-related spectral power in EEG during repetitive movements: A comparison of methods. Journal of Neuroscience Methods, 186(1), 107-115. doi:https://doi.org/10.1016/j.jneumeth.2009.10.022

Ardekani, B. A., Choi, S. J., Hossein-Zadeh, G. A., Porjesz, B., Tanabe, J. L., Lim, K. O., . . . Begleiter, H. (2002). Functional magnetic resonance imaging of brain activity in the visual oddball task. Brain Res Cogn Brain Res, 14(3), 347-356.

Basar, E., Basar-Eroglu, C., Demiralp, T., \& Schürmann, M. (1995). Time and frequency analysis of the brain's distributed gamma-band system. IEEE Engineering in Medicine and Biology, 14, 400-410. doi:10.1109/51.395322

Bassett, D. S., Yang, M., Wymbs, N. F., \& Grafton, S. T. (2015). Learning-induced autonomy of sensorimotor systems. Nat Neurosci, 18(5), 744-751. doi:10.1038/nn.3993

Belouchrani, A., Abed-Meraim, K., Cardoso, J., \& Moulines, E. (1997). A blind source separation technique using second-order statistics. IEEE Transactions on Signal Processing, 45(2), 434-444. doi:10.1109/78.554307

Blatow, M., Rozov, A., Katona, I., Hormuzdi, S. G., Meyer, A. H., Whittington, M. A., . . . Monyer, H. (2003). A novel network of multipolar bursting interneurons generates theta frequency oscillations in neocortex. Neuron, 38(5), 805-817.

Clark, V. P., Fannon, S., Lai, S., Benson, R., \& Bauer, L. (2000). Responses to rare visual target and distractor stimuli using event-related fMRI. J Neurophysiol, 83(5), 3133-3139.

doi:10.1152/jn.2000.83.5.3133

Cohen, J. E., Morin, T. M., \& Stern, C. E. (2018). Theta oscillations increase at critical junctures of overlapping mazes. Paper presented at the CNS2018, Boston.

Dasgupta, S., Sheehan, T. C., Stevens, C. F., \& Navlakha, S. (2018). A neural data structure for novelty detection. Proc Natl Acad Sci U S A, 115(51), 13093-13098.

doi:10.1073/pnas.1814448115

Flint, A. C., \& Connors, B. W. (1996). Two types of network oscillations in neocortex mediated by distinct glutamate receptor subtypes and neuronal populations. J Neurophysiol, 75(2), 951957. doi:10.1152/jn.1996.75.2.951

Friedman, D., Cycowicz, Y. M., \& Gaeta, H. (2001). The novelty P3: an event-related brain potential (ERP) sign of the brain's evaluation of novelty. Neurosci Biobehav Rev, 25(4), 355-373.

Gur, R. C., Turetsky, B. I., Loughead, J., Waxman, J., Snyder, W., Ragland, J. D., . . Gur, R. E. (2007). Hemodynamic responses in neural circuitries for detection of visual target and novelty: An event-related fMRI study. Hum Brain Mapp, 28(4), 263-274. doi:10.1002/hbm.20319 
Jacobs, J., Kahana, M. J., Ekstrom, A. D., \& Fried, I. (2007). Brain Oscillations Control Timing of Single-Neuron Activity in Humans. The Journal of Neuroscience, 27(14), 3839-3844. doi:10.1523/jneurosci.4636-06.2007

Jeewajee, A., Lever, C., Burton, S., O'Keefe, J., \& Burgess, N. (2008). Environmental novelty is signaled by reduction of the hippocampal theta frequency. Hippocampus, 18(4), 340-348. doi:10.1002/hipo.20394

Joyce, C. A., Gorodnitsky, I. F., \& Kutas, M. (2004). Automatic removal of eye movement and blink artifacts from EEG data using blind component separation. Psychophysiology, 41(2), 313325. doi:10.1111/j.1469-8986.2003.00141.x

Kieh, K., A, Laurens, K. R., Duty, T. L., Forster, B. B., \& Liddle, P. F. (2001). An Event-Related fMRI Study of Visual and Auditory Oddball Tasks. Journal of Psychophysiology, 15, 221-240. doi:10.1027//0269-8803.15.4.221

Lachaux, J.-P., Axmacher, N., Mormann, F., Halgren, E., \& Crone, N. E. (2012). High-frequency neural activity and human cognition: Past, present and possible future of intracranial EEG research. Progress in Neurobiology, 98(3), 279-301. doi:https://doi.org/10.1016/j.pneurobio.2012.06.008

Lagerlund, T. D. (1999). EEG source localization (model-dependent and model-independent methods). In E. Niedermeyer \& F. Lopes Da Silva (Eds.), Electroencephalography: Basic Principles, Clinical Applications, and Related Fields (pp. 809-822). Baltimore, MD: Lippincott, Williams and Wilkins.

Lee, H., Simpson, G. V., Logothetis, N. K., \& Rainer, G. (2005). Phase locking of single neuron activity to theta oscillations during working memory in monkey extrastriate visual cortex. Neuron, 45(1), 147-156. doi:10.1016/j.neuron.2004.12.025

Linden, D. E., Prvulovic, D., Formisano, E., Vollinger, M., Zanella, F. E., Goebel, R., \& Dierks, T. (1999). The functional neuroanatomy of target detection: an fMRI study of visual and auditory oddball tasks. Cereb Cortex, 9(8), 815-823.

Mackert, B. M., Wubbeler, G., Leistner, S., Trahms, L., \& Curio, G. (2001). Non-invasive single-trial monitoring of human movement-related brain activation based on DCmagnetoencephalography. Neuroreport, 12(8), 1689-1692.

Malik, A., \& Amin, H. U. (2017). Visual and Cognitive Fatigue During Learning. In Designing EEG Experiments for Studying the Brain (pp. 123-135). London: Academic Press.

Mohr, H., Wolfensteller, U., Betzel, R. F., Misic, B., Sporns, O., Richiardi, J., \& Ruge, H. (2016). Integration and segregation of large-scale brain networks during short-term task automatization. Nat Commun, 7, 13217. doi:10.1038/ncomms 13217

Peng, W., Hu, L., Zhang, Z., \& Hu, Y. (2012). Causality in the association between P300 and alpha event-related desynchronization. PLoS One, 7(4), e34163. doi:10.1371/journal.pone.0034163 
Peng, W., Hu, Y., Mao, Y., \& Babiloni, C. (2015). Widespread cortical alpha-ERD accompanying visual oddball target stimuli is frequency but non-modality specific. Behav Brain Res, 295, 71-77. doi:10.1016/j.bbr.2015.04.051

Perez-Gonzalez, D., Malmierca, M. S., \& Covey, E. (2005). Novelty detector neurons in the mammalian auditory midbrain. Eur J Neurosci, 22(11), 2879-2885. doi:10.1111/j.14609568.2005.04472.x

Pfurtscheller, G., Neuper, C., \& Mohl, W. (1994). Event-related desynchronization (ERD) during visual processing. Int J Psychophysiol, 16(2-3), 147-153.

Raghavachari, S., Lisman, J. E., Tully, M., Madsen, J. R., Bromfield, E. B., \& Kahana, M. J. (2006). Theta oscillations in human cortex during a working-memory task: evidence for local generators. J Neurophysiol, 95(3), 1630-1638. doi:10.1152/jn.00409.2005

Rainer, G., Lee, H., SIMpson, G., \& Logothetis, N. K. (2004). Working-memory related theta $(4 ? 7 \mathrm{~Hz})$ frequency oscillations observed in monkey extrastriate visual cortex. 58-60, 965-969. doi:10.1016/S0925-2312(04)00158-4

Ranganath, C., \& Rainer, G. (2003). Neural mechanisms for detecting and remembering novel events. Nat Rev Neurosci, 4(3), 193-202. doi:10.1038/nrn1052

Schultz, W. (1998). Predictive Reward Signal of Dopamine Neurons. Journal of Neurophysiology, 80(1), 1-27. doi:10.1152/jn.1998.80.1.1

Shine, J. M. (2019). Neuromodulatory Influences on Integration and Segregation in the Brain. Trends Cogn Sci, 23(7), 572-583. doi:10.1016/j.tics.2019.04.002

Shine, J. M., Bissett, P. G., Bell, P. T., Koyejo, O., Balsters, J. H., Gorgolewski, K. J., . . . Poldrack, R. A. (2016). The Dynamics of Functional Brain Networks: Integrated Network States during Cognitive Task Performance. Neuron, 92(2), 544-554. doi:10.1016/j.neuron.2016.09.018

Silva, L. R., Amitai, Y., \& Connors, B. W. (1991). Intrinsic oscillations of neocortex generated by layer 5 pyramidal neurons. Science, 251(4992), 432-435.

Sokolov, E. N. (1963). Perception and the Conditioned Reflex. Oxford: Pergamon Press.

Sutherland, M. T., \& Tang, A. C. (2006). Reliable detection of bilateral activation in human primary somatosensory cortex by unilateral median nerve stimulation. Neuroimage, 33(4), 1042 1054. doi:10.1016/j.neuroimage.2006.08.015

Sutton, S., Braren, M., Zubin, J., \& John, E. R. (1965). Evoked-potential correlates of stimulus uncertainty. Science, 150(3700), 1187-1188.

Tang, A. C., Liu, J. Y., \& Sutherland, M. T. (2005). Recovery of correlated neuronal sources from EEG: the good and bad ways of using SOBI. Neuroimage, 28(2), 507-519.

doi:10.1016/j.neuroimage.2005.06.062 
Tang, A. C., Pearlmutter, B. A., Malaszenko, N. A., \& Phung, D. B. (2002). Independent components of magnetoencephalography: single-trial response onset times. Neuroimage, 17(4), 1773-1789.

Tang, A. C., Pearlmutter, B. A., Malaszenko, N. A., Phung, D. B., \& Reeb, B. C. (2002). Independent components of magnetoencephalography: localization. Neural Comput, 14(8), 1827-1858. doi:10.1162/089976602760128036

Tang, A. C., Pearlmutter, B. A., Zibulevsky, M., \& Carter, S. A. (2000). Blind source separation of multichannel neuromagnetic responses. Neurocomputing, 32-33, 1115-1120. doi:https://doi.org/10.1016/S0925-2312(00)00286-1

Tang, A. C., Sutherland, M. T., \& McKinney, C. J. (2005). Validation of SOBI components from high-density EEG. Neuroimage, 25(2), 539-553. doi:10.1016/j.neuroimage.2004.11.027

Tang, A. C., Sutherland, M. T., McKinney, C. J., Jing-Yu, L., Yan, W., Parra, L. C., . . Sajda, P. (2006, 16-21 July 2006). Classifying Single-Trial ERPs from Visual and Frontal Cortex during Free Viewing. Paper presented at the The 2006 IEEE International Joint Conference on Neural Network Proceedings. 


\author{
Author Notes \\ Grant funding sources \\ This project is funded by a seed grant from the University of Hong Kong to AC Tang. \\ Acknowledgements \\ We thank Mr. Y.Q. Hua and Drs. G. Ouyang and J. Liu for their assistance. \\ Conflict of interest statement \\ The authors declare no competing financial interests.
}


Table 1. Two distinct stages of novelty processing: the peak latencies and power of theta and alpha frequency bands elicited by novelty in the Early visual component

\begin{tabular}{|c|c|c|c|c|}
\hline \multirow{2}{*}{ Participants } & \multicolumn{2}{|c|}{ Peak latency (ms) } & \multicolumn{2}{|c|}{ Peak power } \\
\hline & Theta & Alpha & Theta & Alpha \\
\hline 1 & 111 & 683 & 1 & -12 \\
\hline 2 & 161 & 623 & 4 & -6 \\
\hline 3 & 237 & 639 & 4 & -7 \\
\hline 4 & 339 & 716 & 6 & -13 \\
\hline 5 & 221 & 606 & 2 & -15 \\
\hline 6 & 262 & 207 & 5 & -3 \\
\hline 7 & 188 & 512 & 2 & -10 \\
\hline 8 & 120 & 635 & 7 & -2 \\
\hline 9 & 130 & 366 & 4 & -4 \\
\hline 10 & 318 & 689 & 3 & -8 \\
\hline 11 & 325 & 485 & 3 & -8 \\
\hline 12 & 54 & 207 & 2 & -4 \\
\hline 13 & 196 & 734 & 6 & -4 \\
\hline Mean & 205 & 546 & 4 & -7 \\
\hline SEM & 24 & 21 & 1 & 1 \\
\hline
\end{tabular}


Table 2: Results of dipole source modeling of the Early visual component

\begin{tabular}{|c|c|c|c|c|c|c|c|c|}
\hline & \multicolumn{3}{|c|}{ Talairach coordinates } & \multirow[b]{2}{*}{ GoF } & \multirow[b]{2}{*}{$\mathbf{R V}$} & \multicolumn{3}{|c|}{ Brain region } \\
\hline & $\mathbf{x}$ & $\mathbf{y}$ & $\mathbf{z}$ & & & Lobe & Cortex & BA \\
\hline 1 & 30 & -85 & 30 & $98 \%$ & $2 \%$ & Occipital Lobe & Cuneus & 19 \\
\hline 2 & 26 & -86 & -2 & $96 \%$ & $4 \%$ & Occipital Lobe & Middle Occipital Gyrus & 18 \\
\hline 3 & 25 & -73 & -6 & $98 \%$ & $2 \%$ & Occipital Lobe & Lingual Gyrus & 19 \\
\hline 4 & 21 & -84 & -5 & $95 \%$ & $5 \%$ & Occipital Lobe & Middle Occipital Gyrus & 18 \\
\hline 5 & 36 & -77 & 37 & $95 \%$ & $5 \%$ & Parietal Lobe & Precuneus & 19 \\
\hline 6 & 8 & -98 & 15 & $95 \%$ & $5 \%$ & Occipital Lobe & Middle Occipital Gyrus & 18 \\
\hline 7 & 31 & -78 & 22 & $92 \%$ & $8 \%$ & Occipital Lobe & Middle Occipital Gyrus & 19 \\
\hline 8 & 12 & -89 & -11 & $95 \%$ & $5 \%$ & Occipital Lobe & Lingual Gyrus & 18 \\
\hline 9 & 33 & -84 & 12 & $94 \%$ & $6 \%$ & Occipital Lobe & Middle Occipital Gyrus & 19 \\
\hline 10 & 27 & -91 & 20 & $94 \%$ & $6 \%$ & Occipital Lobe & Middle Occipital Gyrus & 18 \\
\hline 11 & 29 & -91 & -20 & $94 \%$ & $6 \%$ & Occipital Lobe & Fusiform Gyrus & 18 \\
\hline 12 & 31 & -53 & -7 & $94 \%$ & $6 \%$ & Limbic Lobe & Parahippocampal Gyrus & 19 \\
\hline 13 & 49 & -73 & 2 & $99 \%$ & $1 \%$ & Occipital Lobe & Middle Occipital Gyrus & 19 \\
\hline Mean & 28 & -82 & 7 & $95 \%$ & $5 \%$ & Occipital Lobe & Middle Occipital Gyrus & 18 \\
\hline SEM & 3 & 3 & 5 & 1 & $1 \%$ & & & \\
\hline
\end{tabular}

GoF, goodness of fit; RV, residual variance; BA, Brodmann's area 


\section{Figure captions}

Figure 1. Distinct spatial (scalp projection) and temporal (ERP, time domain) characteristics of the SOBI extracted early visual (Early) and late P300 (Late) components in the individual participants $(\mathrm{N}=13)$.

Red and blue colors in the scalp maps indicate positive or negative polarity. The shaded regions mark the time windows for measuring the peaks in the Early visual and Late P300 components.

Figure 2. Dissociation of two stages of novelty processing: distinctive patterns of power changes across different frequency domains of neural oscillation.

(A and B) power as a function of frequency bands in the Early visual (A) and Late P300 (B) components. Power computed using 1 second window. (C) novelty effect (power difference, Rare-Frequent). *, $\mathrm{p}<0.05$; $^{* *}, \mathrm{p}<0.01$; $^{* *}, \mathrm{p}<0.001 ;$ m.s., $0.05<\mathrm{p}<0.10$; n.s., not significant. Red *, one-sample t-test; black*, paired t test.

Figure 3. Dissociation of two stages of novelty processing: consistent timing differences in peak theta novelty responses between the early visual and late P300 components. Time frequency plots shown for group (A) and a representative individual (B). Frequency range shown is 3 to $40 \mathrm{~Hz}$. Time zero indicates stimulus onset time. The blue vertical line indicates the time of maximum power in the theta band $(4-7 \mathrm{~Hz})$ whereas the red vertical line indicates the time of maximum alpha power $(8-15 \mathrm{~Hz})$. 


\section{Figure 4. Scatterplots from the correlation analyses}

Scatterplots showing the correlation between (A) the latency of Early theta power increase and reaction time of the subject's button press. (B) the latency of Early theta power increases and the latency of Late theta power increases.

\section{Figure 5. Dipole modelling analysis of the early visual components}
(A) current source density maps (group average) of fitted dipole model (GoF: $95 \pm 1 \%, \mathrm{RV}: 5 \pm$ 1\%). (B) solutions of equivalent dipole modelling superimposed on the sagittal, horizontal, and coronal structural MRI of a standard brain (BESA). In the MRI slides, only 8 pairs of equivalent dipoles were shown in the horizontal (z-axis) slide and 9 pairs in the coronal (y-axis) slide because some of the dipoles were out of range in both planes at the current level. 
Figure 1

Figure 1. Distinct spatial (scalp projection) and temporal (ERP, time domain) characteristics of the SOBI extracted early visual (Early) and late P300 (Late) components in the individual participants $(\mathrm{N}=13)$. Red and blue colors in the scalp maps indicate positive or negative polarity. The shaded regions mark the time windows for measuring the peaks in the Early visual and Late P300 components. 
Figure 2. Dissociation of two stages of novelty processing: distinctive patterns of power changes across different frequency domains of neural oscillation.

( $A$ and $B$ ) power as a function of frequency bands in the Early visual $(A)$ and Late P300 (B) components. Power computed using 1 second window. (C) novelty effect (power difference, Rare-Frequent). $*, p<0.05$; $* *, \mathrm{p}<0.01$; ***, $\mathrm{p}<0.001$; m.s., $0.05<\mathrm{p}<0.10 ;$ n.s., not significant. Red *, one-sample t-test; black *, paired t test.
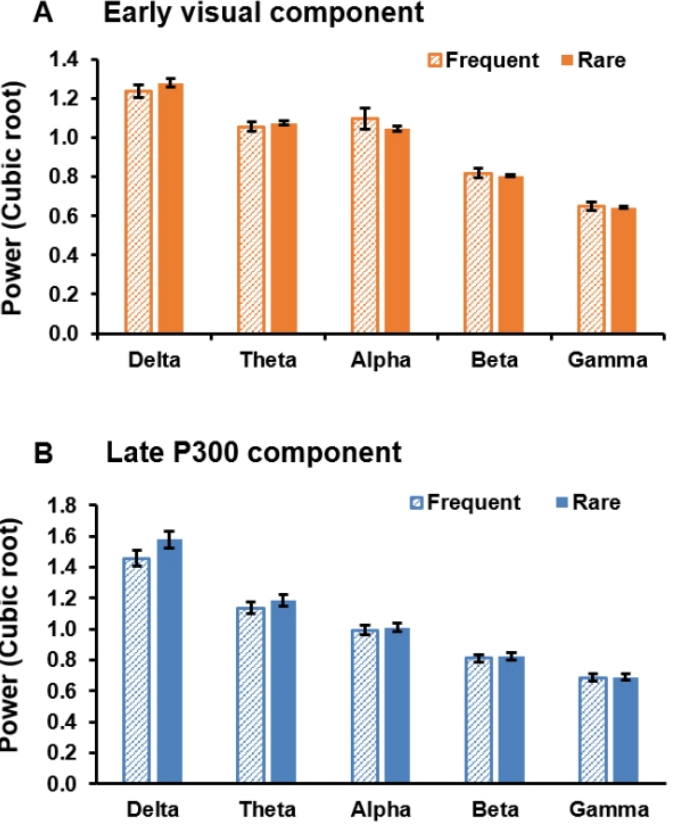

C Novelty effect (Rare - Frequent)

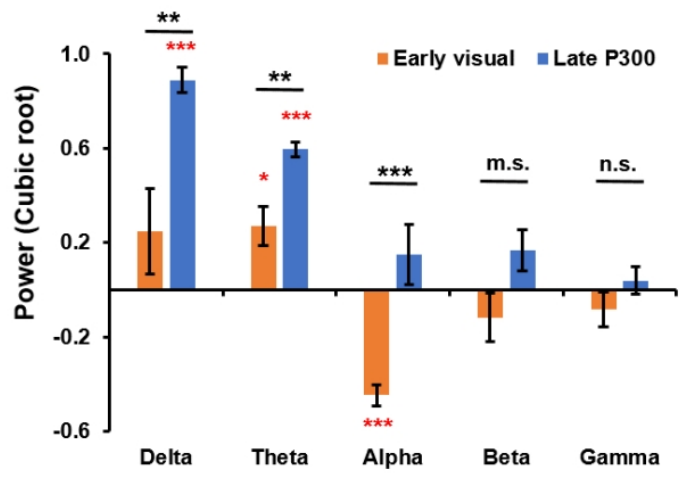


Figure 3

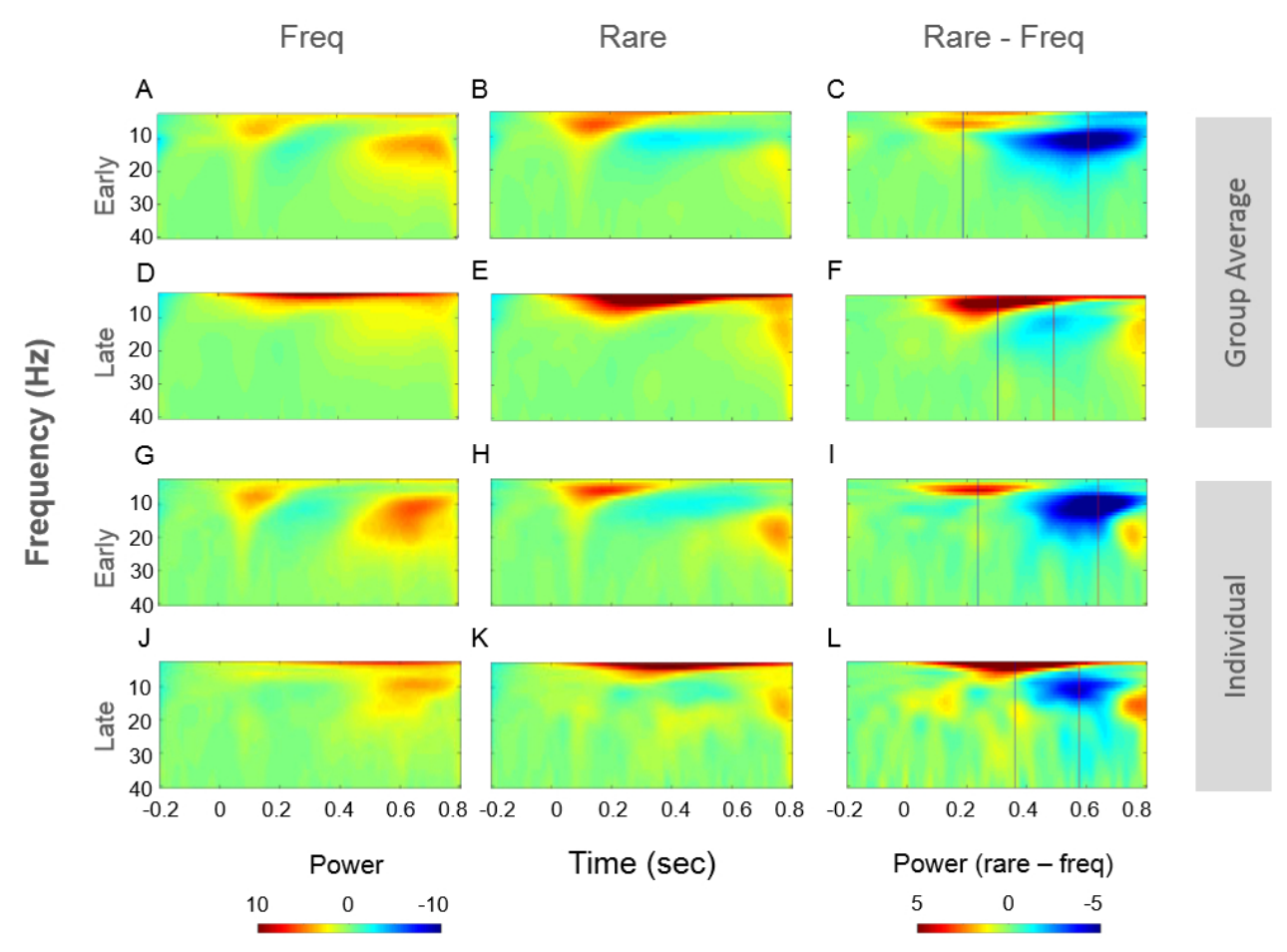

Figure 3. Dissociation of two stages of novelty processing: consistent timing differences in peak theta novelty responses between the early visual and late P300 components.

Time frequency plots shown for group (A) and a representative individual (B). Frequency range shown is 3 to $40 \mathrm{~Hz}$. Time zero indicates stimulus onset time. The blue vertical line indicates the time of maximum power in the theta band $(4-7 \mathrm{~Hz})$ whereas the red vertical line indicates the time of maximum alpha power (8- $15 \mathrm{~Hz})$. 


\section{Figure 4}

\section{A}

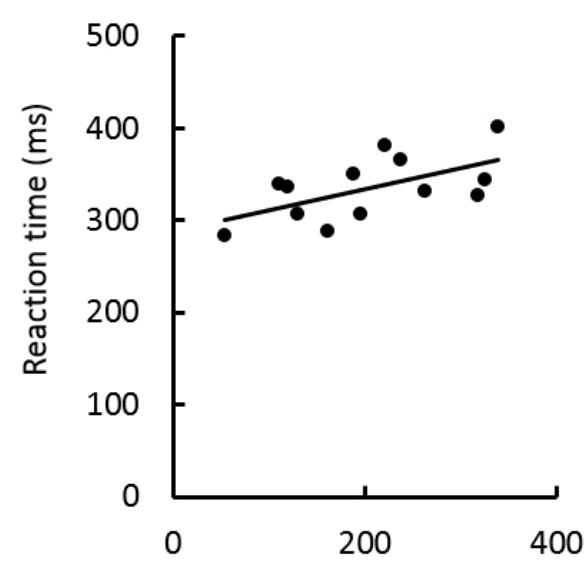

B

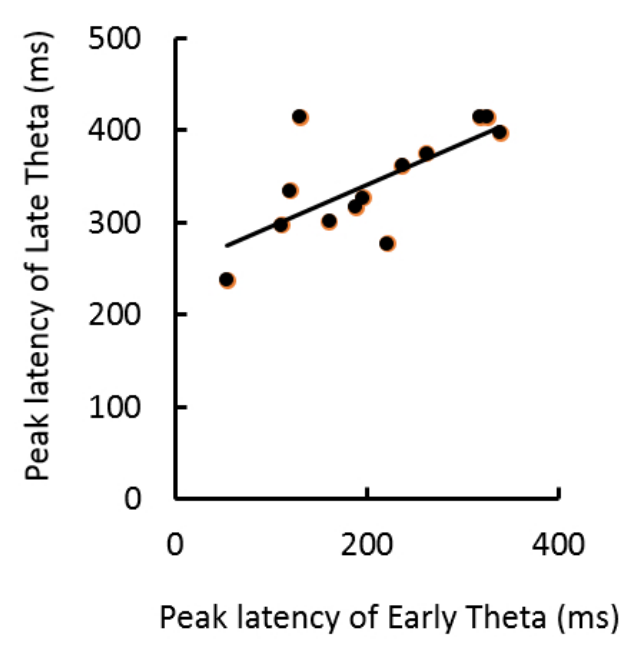

Figure 4. Scatterplots from the correlation analyses

Scatterplots showing the correlation between $(A)$ the latency of Early theta power increase and reaction time of the subject's button press. (B) the latency of Early theta power increases and the latency of Late theta power increases. 


\section{Figure 5}
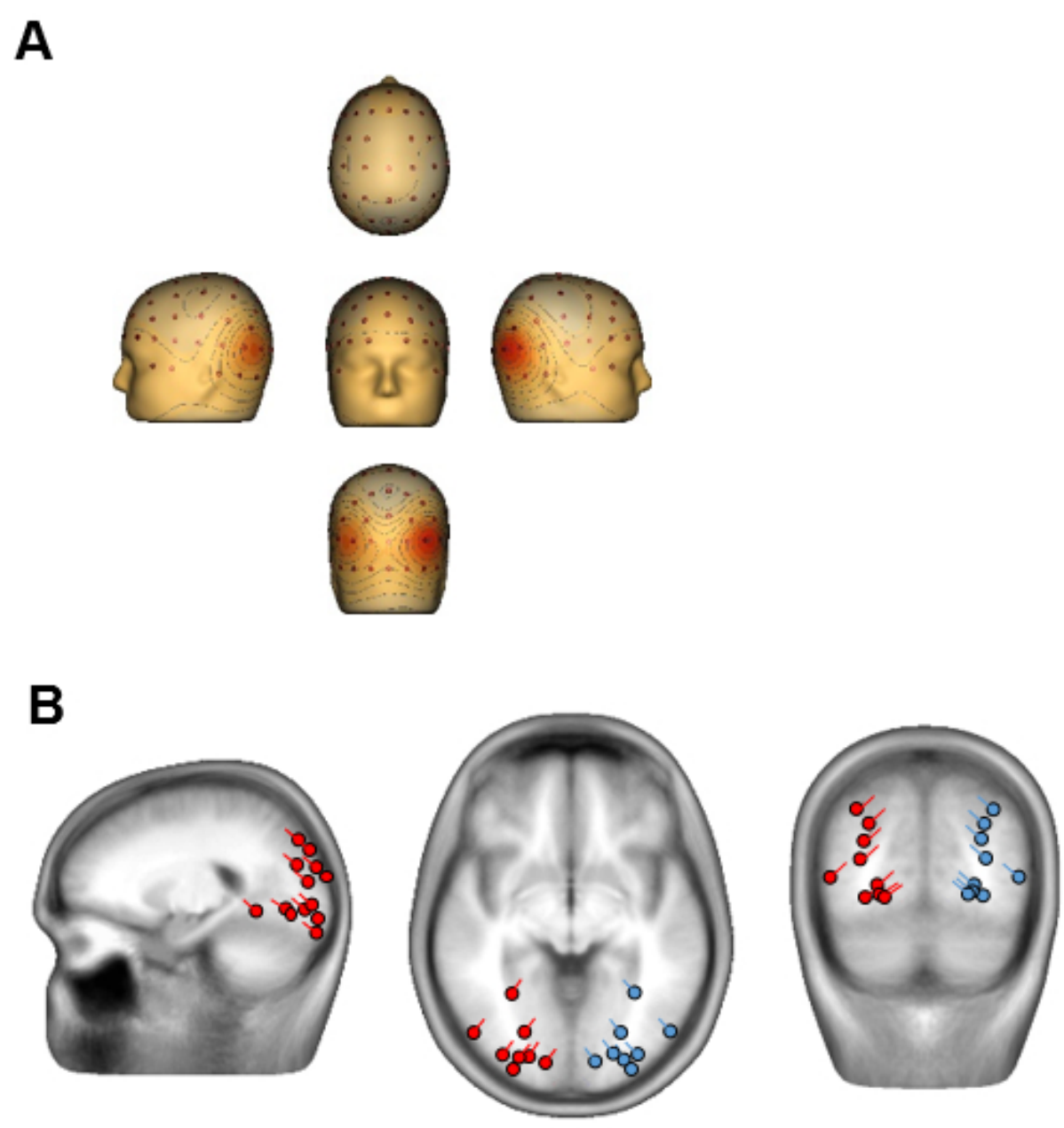

Figure 5. Dipole modelling analysis of the early visual components

(A) current source density maps (group average) of fitted dipole model (GoF: $95 \pm 1 \%, \mathrm{RV}: 5 \pm 1 \%$ ). (B) solutions of equivalent dipole modelling superimposed on the sagittal, horizontal, and coronal structural MRI of a standard brain (BESA). In the MRI slides, only 8 pairs of equivalent dipoles were shown in the horizontal ( $z$-axis) slide and 9 pairs in the coronal ( $y$-axis) slide because some of the dipoles were out of range in both planes at the current level. 
Supplementary Figure 1

Early Visual Component
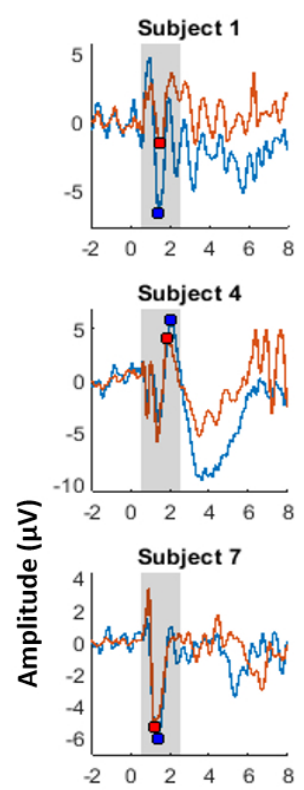

Subject 10
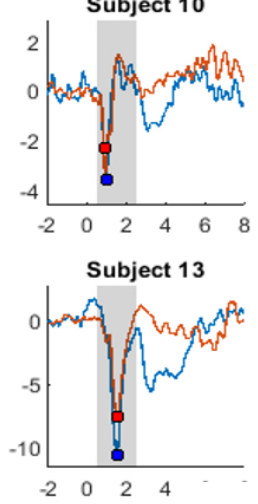
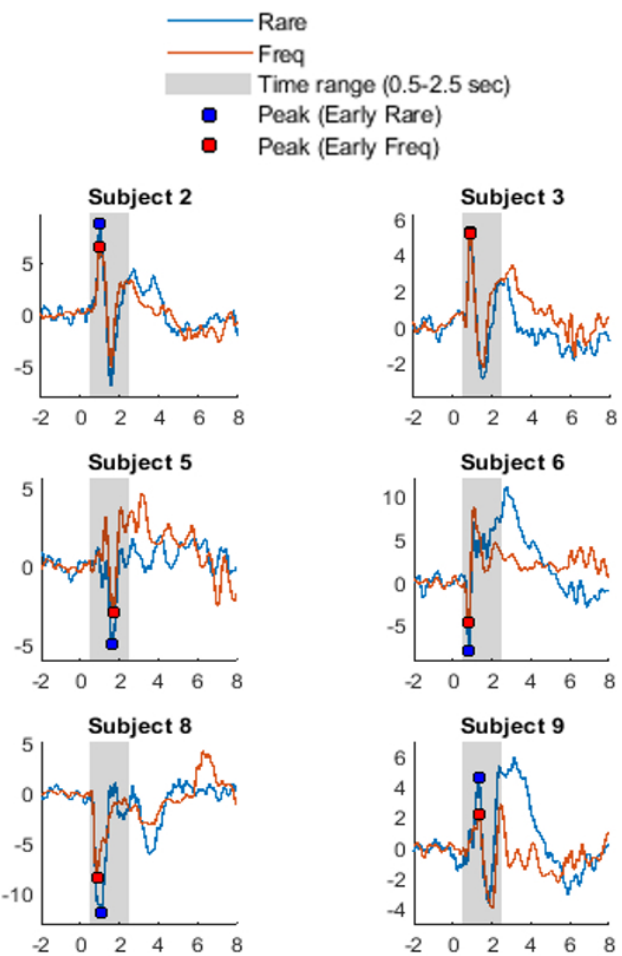

Subject 11
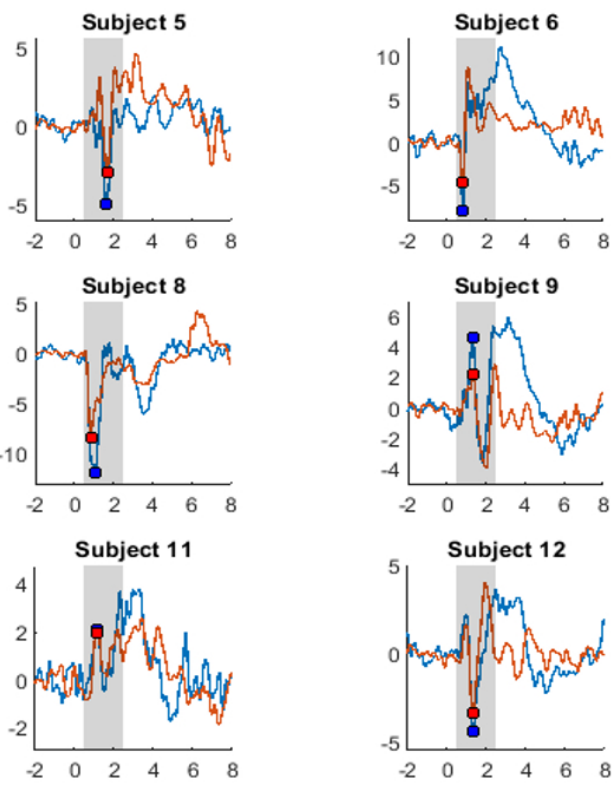

Subject 9
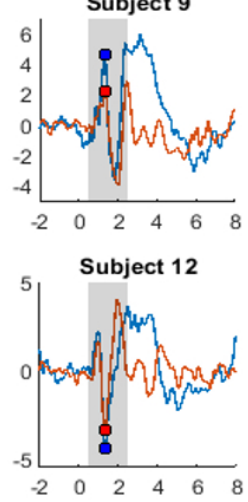

Time (sec) 
Supplementary Figure 2
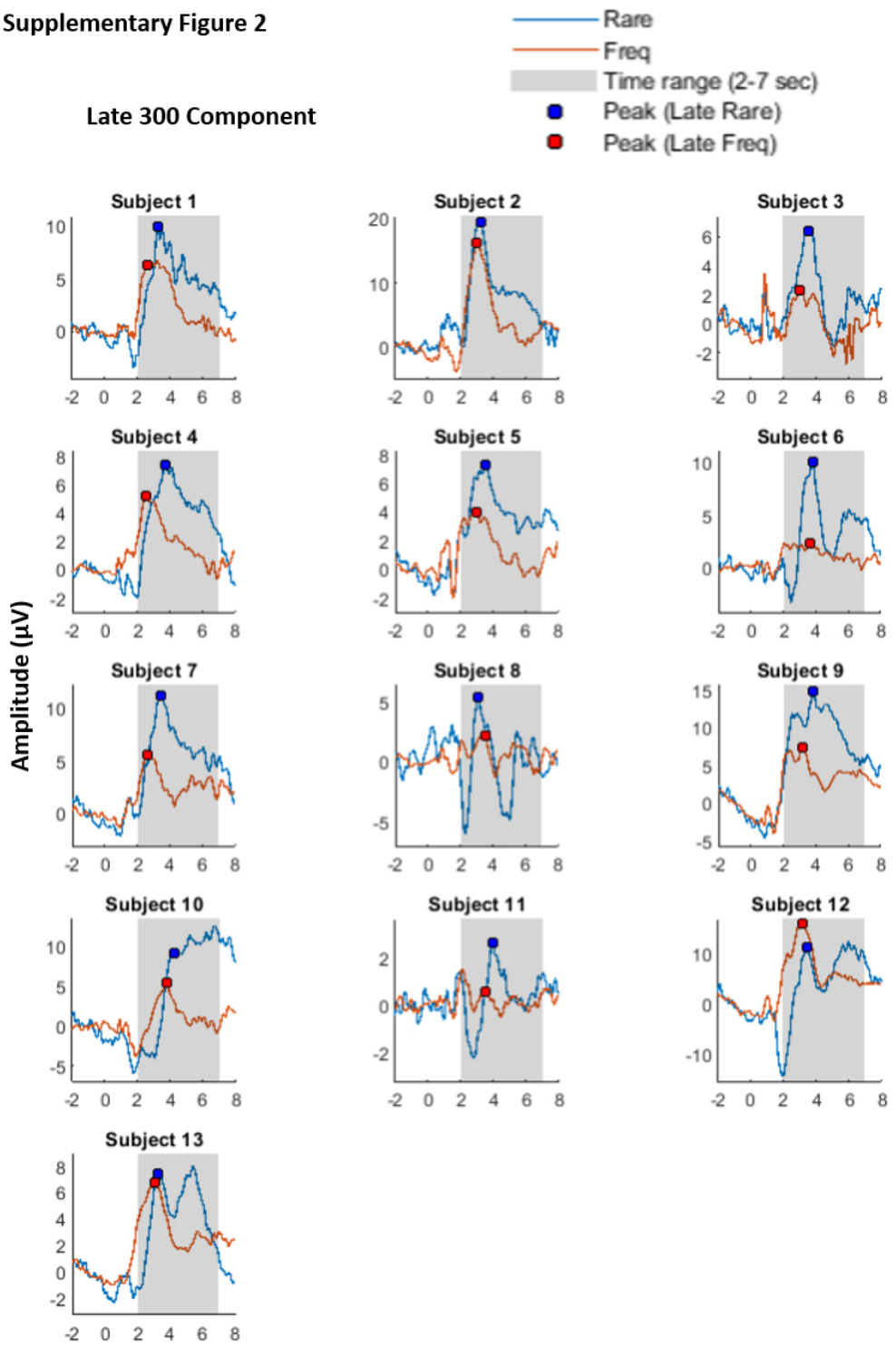

Time (sec) 